\title{
Mycophenolic acid (MPA) modulates host cellular autophagy progression in sub genomic dengue virus-2 replicon cells
}

\author{
Nageswar Reddy Manchala ${ }^{\mathrm{a}}$, Ranjeet Dungdung ${ }^{\mathrm{a}}$, Pankaj Trivedi ${ }^{\mathrm{b}}$, \\ Unnikrishnan Unniyampurath ${ }^{c}$, Rajendra Pilankatta ${ }^{a, *}$ \\ ${ }^{a}$ Department of Biochemistry and Molecular Biology, School of Biological Sciences, Central University of Kerala, Tejaswini Hills, Periye, 671320, Kerala, India \\ ${ }^{\mathrm{b}}$ Department of Experimental Medicine, Sapienza University, 00161, Rome, Italy \\ ${ }^{\mathrm{c}}$ Program on Emerging Infectious Diseases, Duke-NUS Medical School, 8 College Road, Singapore, 169857
}

\section{A R T I C L E I N F O}

\section{Keywords:}

Dengue

Autophagy

Viruses

Replicon

MPA

Replication

LC3 II

p62

\begin{abstract}
A B S T R A C T
Cellular autophagy (Macrophagy) is a self-degradative process, executed through the network of autophagy associated genes (ATGs) encoded proteins. Both in vitro and in vivo studies suggest that dengue virus (DENV) induces autophagy and supports the viral genome replication and translation. Therefore, the cellular autophagy induced by dengue virus can be a good target for antiviral drug development. The action of mycophenolic acid (MPA), a specific inhibitor of DENV replication, was investigated in the stable BHK-21/DENV2 replicon cells. The inhibition was mediated by enhanced degradation of autophagic substrates in stable BHK-21/DENV2 replicon cells as evidenced by a decrease in lapidated LC3 (LC3II) and p62 expression in the presence of MPA. In contrast, the results indicated that four gene sets, namely Transmembrane protein 74 (TMEM74), Unc-51-like kinase 2 (ULK2), Cathepsin D (CTSD) and Estrogen receptor 1 (ESR1) were upregulated in stable BHK-21/ DENV2 replicon cells, due to the sustained dynamic replication of DENV2 genome. These ATGs involved in the pre-autophagosomal structure (PAS) formation, were suppressed in the presence MPA. Instead, MPA induced the expression of different set of autophagy genes such as ATG4, AKT1, APP, ATG16L1, ATG16L2, B2M and HPRT1. An enzyme involved in the nucleotide salvage pathway, HPRT1, was highly expressed in the presence of MPA. The study shows that DENV2 replication is dependent on PAS formation and is inhibited in the presence of MPA by enhancing the degradation of autophagic substrates and suppression of PAS formation. This study provides impetus in designing MPA analogues to effectively inhibit dengue viral replication.
\end{abstract}

\section{Introduction}

Dengue is a major public health concern that causes severe morbidity and mortality, especially in tropical and subtropical countries. Dengue fever is an Aedes mosquito vector-borne ailment caused by any one of four serotypes of dengue virus (DENV 1 to 4) [1]. DENV genome consists of a single positive-strand RNA and replicates efficiently in the Endoplasmic Reticulum (ER) of the infected cells by hijacking host autophagy machinery [2]. Autophagy is an evolutionarily conserved protective mechanism that is induced during DENV infection. It is involved in shuttling the damaged cellular cargo to lysosomes for degradation and recycling [3]. This complex process is coordinated by proteins encoded by the autophagy-associated genes (ATGs) [4]. Autophagy is required for optimal RNA replication of DENV $[5,6]$ and the infection could induce autophagy in in vivo models [7].

RNA viruses such as DENVs induce autophagy to get supportive

\footnotetext{
Abbreviations: ATG, autophagy; ATGs, autophagy associated genes; BHK, baby hamster kidney cells; oC, degree centigrade; C, capsid protein; cDNA, complementary

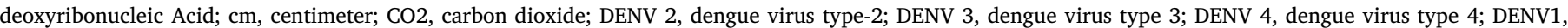

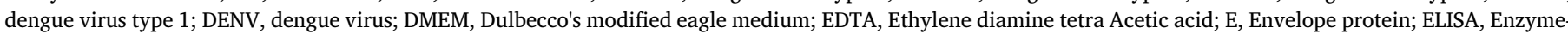

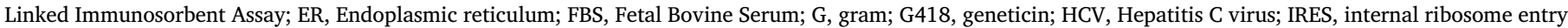

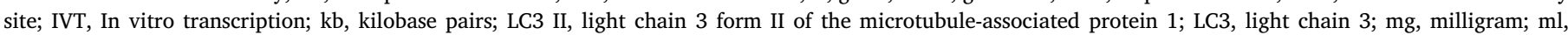

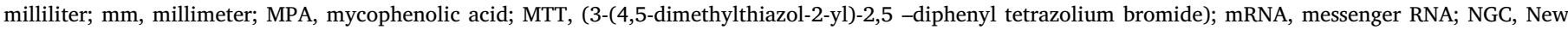

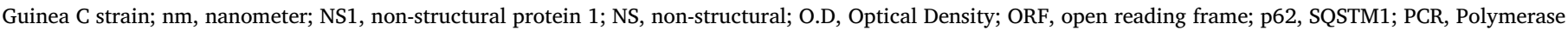

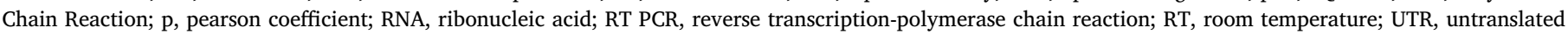
region

* Corresponding author.

E-mail address: praj74@cukerala.ac.in (R. Pilankatta).
} 
benefits for their replication using ER membrane scaffolds [8]. DENV infection causes ER membrane remodeling in an infected cell, controlled by a large number of autophagic proteins and its upstream/ downstream signaling molecules. Autophagy genes play antiviral and/ or proviral role in the life cycle and pathogenesis of many different viruses [9]. Also, it was observed that the autophagy helps to maintain homeostatic environment in DENV infected cells and favors for the production of virions [10,11]. The RNA viruses subvert autophagy machinery to establish its replication within the host cells [8]. DENV utilizes autophagy to foster its cellular growth and non-lytic cellular egress [12]. Many postulates suggest that inhibition of autophagy might reduce the viral replication by reducing number of virions and protection from cellular apoptosis [13].

Inhibitors such as Bafilomycin A1, Chloroquine, and Pepstatin A/ E64d inhibit degradation of the autolysosome contents via inhibition of the $\mathrm{Na}+/ \mathrm{H}+$ pump at the lysosome, increasing lysosomal $\mathrm{pH}$ and inhibiting acidic lysosomal proteases, respectively. The earlier studies have shown that the nucleoside analogues such as 2'-C-methyladenosine $($ EC50 $=2.42 \pm 0.59 \mu \mathrm{M})$, and antiviral drugs like Ribavirin $(\mathrm{EC} 50=6.77 \pm 1.33 \mu \mathrm{M})$, and MPA $($ EC50 $=1.31 \pm 0.27 \mu \mathrm{M})$ can effectively inhibit DENV replication [14,15]. MPA inhibits DENV genome replication, antigen expression, and maturation [16]. The proposed mechanism of MPA action suggests that it inhibits Inosine monophosphate dehydrogenase (IMPD) enzyme and thereby cause depletion of the intracellular Guanosine-5'-triphosphate (GTP) pool [15]. Although, some progress has been made in understanding how autophagy genes are regulated by dengue viral replication during infection [9], very little is known on the identity of dengue viral proteins involved in the host autophagy induction and maturation. It is also not clear whether DENV induces the autophagy at an early stage or later stage (egress) of virus life cycle.

Baby Hamster Kidney (BHK-21) cells are permissive cellular model for many viruses including DENV [17]. Therefore, we sought to investigate the autophagy related gene (ATG) expression in stable BHK21/DENV2 replicon cells. The subgenomic replicon encodes DENV2 non-structural genes involved in the dengue viral replication. The stable replicon system is broadly used in the studies of trans-complementation, adaptive mutation, viral assembly and packaging of Kunjin, Yellow fever and West Nile viruses [14]. In the present study, we investigated the effect of MPA on the modulation of host autophagy gene expression during dengue virus RNA replication in stable BHK 21/ DENV2 replicon cells along with assessment of the degradation of autophagic substrates such as LC3 and p62.

\section{Materials and methodology}

\subsection{Cells}

Baby Hamster Kidney (BHK-21) cells were procured from National Centre for Cell Science, Pune, India. Dulbecco's modified Eagle medium (Himedia; catalogue\#AT149), FBS (Life Technologies; catalogue\#10439-016), L- Glutamine (Sigma; catalogue\#G7513), Penicillin-Streptomycin (Sigma; catalogue\#P4333), mycophenolic acid (Sigma; catalogue\#M5255) an inhibitor of DENV-RNA replication, were procured from the supplier (Sigma-Aldrich ${ }^{\circledR}$ ). The cells were cultured and maintained at $37{ }^{\circ} \mathrm{C}$ with $5 \% \mathrm{CO}_{2}$ incubator (Thermo Scientific $^{\mathrm{TM}}$ Forma $^{\mathrm{TM}}$ Steri-Cycle i160).

The pRS424 plasmid containing frame work of non-structural genes of DENV 2 (NGCstrain) was a kind gift from Prof. Padmanabhan's laboratory (Georgetown University, Washington DC, USA). The pRS424 plasmid containing coding regions (RLuc-IRES-Neo ${ }^{\mathrm{r}}$-DENV2-NS) were cloned downstream of SP6 promoter. The gene order of replicon is $5^{\prime}$ UTR, N terminal coding sequence of capsid (C), Renilla Luciferase reporter (Rluc) with translational termination codon and internal ribosome entry site (IRES) for 5'-cap-independent translation of the downstream ORF that encodes a poly protein precursor $\mathrm{C}$ terminus $\mathrm{E}$ -
NS1-NS2A, NS2B, NS4A, NS4B, NS5 followed by 3' UTR.

\subsection{In vitro transcription (IVT) of DENV2 subgenomic replicon cDNA}

The pRS424 plasmid encoding DENV2 replicon cDNA was propagated in Stbl2 competent cells (Thermo Fisher Scientific; catalogue \#10268019) and was linearized by Sac I restriction enzyme digestion and purified with PCR purification kit (Thermo Fisher Scientific; catalogue \#K310001). Further, the linearized plasmid was used as a template for in vitro RNA synthesis and DENV 2 subgenome was transcribed by SP6 polymerase using in vitro transcription kit (MEGA script; AM1330, Invitrogen) as per the manufacturer's instructions. Finally IVT-RNA was prepared.

\subsection{Transfection of IVT-RNA into naïve BHK-21 cells and generation of stable BHK-21/DENV2 replicon cells}

The newly synthesized IVT-RNA was resolved on agarose gel (2\%) and purified from the gel as per the instructions of gel purification kit (Qiagen, catalogue \# 28704). The gel purified RNA was transfected into naïve BHK-21 cells using transfection kit (Mirus Bio Trans IT-mRNA; MIR 2225). The transfected BHK 21 cells were grown in DMEM containing Geneticin (G418) selectable marker. Finally the resistant, stable BHK-21/DENV2 clones were selected under G418 selection at the concentration of $400 \mu \mathrm{g} / \mathrm{ml}$. The stable BHK 21 replicon cells were maintained at $(37 \pm 1){ }^{\circ} \mathrm{C}$ in $5 \% \mathrm{CO}_{2}$ in $50-60 \%$ confluence by sub culturing every 3-4 days.

\subsection{Characterization of stable BHK-21/DENV2 replicon cells}

\subsubsection{DENV2- NS4A gene amplification by reverse transcriptase PCR (RT-} PCR)

Total RNA was isolated from the stable BHK-21/DENV2 replicon cells as well as naive BHK-21 cells using TRIzol ${ }^{\mathrm{TM}}$ reagent (Invitrogen, catalogue\#15596018). The reverse transcriptase enzyme was added to the purified RNA ( $1 \mu \mathrm{g}$ concentration) and the mixture was incubated at $42{ }^{\circ} \mathrm{C}$ for 15 minutes and $95^{\circ} \mathrm{C}$ for 5 minutes to convert total RNA into cDNA in an independent reactions as per RT-PCR kit manufacturer's instruction (Qiagen Ltd, West Sussex, UK; , catalogue \#205311). DENV2- NS4A gene was amplified under the conditions of initial denaturation at $94^{\circ} \mathrm{C}$ for 2 minutes and 35 cycles of denaturation at $94^{\circ} \mathrm{C}$ for 30 seconds, annealing at $53^{\circ} \mathrm{C}$ for 30 seconds and elongation at $72{ }^{\circ} \mathrm{C}$ for 60 seconds and final extension of $72^{\circ} \mathrm{C}$ for 7 minutes from cDNA of respective samples by specific DENV2-NS4A sense and anti sense primers. The amplified DNA fragments were resolved and confirmed on agarose gel (2\%). As an internal control Beta actin gene transcript was amplified by RT-PCR.

\subsubsection{Renilla luciferase (RLuc) enzyme assay}

The stable BHK-21/DENV2 replicon cells were incubated in the presence of $1.25 \mu \mathrm{M}$ MPA for 24 hours. Renilla luciferase (RLuc) enzyme activities in $20 \mu \mathrm{L}$ stable BHK-21/DENV2 replicon cell lysate (untreated group) and MPA-treated stable BHK-21/DENV2 replicon cell lysate (treated group) were quantified independently by RLuc enzyme assay. The assay was performed in three independent experiments $(n=3)$. The experimental procedure was performed as per the manufacturer's instructions (Promega Corporation, Madison, WI, USA; catalogue \#E2810). RLuc enzyme activities were measured in the cell lysates and the statistical analysis was used to assess the difference between the untreated group (without drug) and treated group (with the drug). The difference in RLuc enzyme activity in treated versus untreated group is detected by luminescence signals in a multimode plate reader (PerkinElmer, part number\#2300-000 M). The values were submitted to t-test and the bar diagram was made using graph pad prism software, version 5.0. The values of $\mathrm{p}<0.05$ were considered statistically significant. 


\subsubsection{Detection of the secreted DENV2- NS1 protein by ELISA}

The stable BHK-21/DENV2 replicon cells were incubated in the presence of $1.25 \mu \mathrm{M}$ MPA for 24 hours. The presence of secreted DENV2- NS1protein in $100 \mu$ culture supernatant of stable BHK-21/ DENV2 replicon cell (untreated group) and MPA-treated stable BHK21/DENV2 replicon cells (treated group) was examined independently $(\mathrm{n}=3$ ) by DENV2- NS1 micro ELISA detection kit (J. Mitra \& Co, India). The kit is based on a single-step sandwich immunoassay to detect DENV2- NS1 protein in cell culture supernatant. The kit contains monoclonal antibody coated on micro wells, which can detect dengue viral NS1 protein. Normal DMEM used as mock control and standard NS1protein used as a positive control (provided with the kit). The optical density (O.D) of DENV2-NS1 protein was measured spectophotometrically at $450 \mathrm{nms}$ in the above culture supernatants of treated and untreated group. The values were submitted to t-test and bar graph was plotted using graph pad prism software, version 5.0. The values of $\mathrm{p}<0.05$ were considered statistically significant.

\subsection{Assessment of the effect of MPA on autophagic flux in stable BHK-21/ DENV2 replicon cells}

\subsubsection{Cytotoxic effect of MPA by MTT assay}

The stable BHK-21/DENV2 replicon cells were seeded in 96-well flat-bottomed plate (Nunc, Nunclon ${ }^{\mathrm{TM}}$ Surface Roskilde, Denmark) @ $1 \times 10^{4}$ cells per well in DMEM with $10 \%$ FBS. The cells were exposed to MPA range from 0 to $5 \mu \mathrm{M}$ for $24 \mathrm{~h}$. The cytotoxic effect of MPA on stable BHK-21/DENV2 replicon cells was determined using MTT (Sigma-Aldrich; catalogue\# CT01-5) assay by adding $20 \mu \mathrm{l}$ of MTT $(5 \mathrm{mg} / \mathrm{ml})$ reagent to each well. The cells were incubated for 4 hours at $37^{\circ} \mathrm{C}$ in $5 \% \mathrm{CO}_{2}$. Then, DMEM containing MTT was removed from the wells and newly formed Formazan crystals were dissolved in $100 \mu \mathrm{l}$ Dimethyl sulfoxide (DMSO). Within 30 minutes, the absorbance was measured at $570 \mathrm{~nm}$ using Enspire multimode micro plate reader (PerkinElmer). The cell viability (\%) of stable BHK-21/DENV2 replicon cells as well as MPA treated cells was calculated. The optimum concentration of MPA was determined by plotting the dose response curve.

\subsubsection{Total cellular RNA isolation}

Total cellular RNA was extracted from stable BHK-21/DENV2 replicon cells (Group1), MPA treated cells (Group 2) and control group (Naïve BHK-21 cells) using RNeasy mini kit (Qiagen, catalogue\#74106). The genomic DNA contamination was removed from the samples by treating with RNase-free DNase (provided with kit) for 15 minutes at room temperature. The concentration of RNA was measured by a UV Vis spectrophotometer (NanoDrop 2000, Thermo Fisher, USA). The presence of RNA was confirmed on $2 \%$ agarose gels.

\subsection{3. cDNA synthesis and confirmation}

The cellular RNA was used as a template for complementary DNA (cDNA) synthesis using RT-PCR kit as per the manufacturer (Qiagen, catalogue\#205310). The cDNA was synthesized by incubating at $25^{\circ} \mathrm{C}$ for 10 minutes, $42^{\circ} \mathrm{C}$ for 60 minutes and at $70{ }^{\circ} \mathrm{C}$ for 15 minutes. The concentration of each cDNA prepared from Group 1, Group 2 and control group were measured using UV Vis spectrophotometer. The PCR amplified products were confirmed on $1 \%$ agarose gel. The beta actin gene amplified product was used as an internal control.

\subsubsection{Autophagy gene expression analysis by quantitative real time RT-} $P C R$

The human RT2 Profiler PCR array plates (PAHS-084Z, from SABiosciences, USA) in 96-well format were used for the analyzing the expression of 84 autophagy genes in a separate array plates in stable BHK-21/DENV2 replicon cells, MPA-treated stable BHK-21/DENV2 replicon cells and control group. Each gene was analyzed in duplicate for each group (total six array plates). Using RT2 profiler kit, one $\mu \mathrm{g}$ of total RNA obtained from the above was treated with genomic DNA elimination mixture (provided with kit) by incubating at $45^{\circ} \mathrm{C}$ for 5 minutes. The reaction mixture was incubated at $42^{\circ} \mathrm{C}$ for 15 minutes and then $95^{\circ} \mathrm{C}$ for 5 minutes to convert RNA into cDNA. For each plate the master mix was prepared as per the manufacturer's instructions and $25 \mu \mathrm{L}$ reaction volume was loaded in each well of 96 well plate. The real time RT-PCR was performed using RT $^{2}$ PCR SYBR Green master mix. The array plate was covered with seal and kept in Light cycler 480 (Roche Diagnostics Ltd, Burgess Hill, UK) and the program was set up according to manufacturer's instructions.

The relative expression ratio $(R)$ of target genes normalized with control group in both stable BHK-21/DENV2 replicon cells (Group 1) and MPA treated stable BHK-21/DENV2 replicon cells (Group 2) were calculated. The amplification data (fold changes in the threshold cycle (Ct) values of all the genes) was analyzed by the $\Delta \Delta \mathrm{Ct}$ method for determining gene expression changes with the following formula: $\mathrm{R}=2^{\wedge \mathrm{CT}}$ (target) $/ 2^{\wedge \mathrm{CT}}($ ref), with CT(target) $=(\mathrm{CT}$ target $-\mathrm{CT}$ control) of target genes and CT(ref) $=$ (CT ref - CT control) of internal control genes. After finishing the last cycle, the melt curve (Tm) was performed at $60^{\circ} \mathrm{C}, 15 \mathrm{~s}$ and $95^{\circ} \mathrm{C}$ continuous mode.

\subsubsection{Statistical analysis of $q$ RT-PCR data}

The fold changes in the $\mathrm{Ct}$ value of all the genes were grouped into 3 sets: stable BHK-21/DENV2 replicon cells (Group 1) and MPA treated BHK-21/DENV2 replicon cells (Group 2) and control group (Naïve BHK -21 cells). The Ct values of all the genes were entered in Microsoft excel spreadsheet and analyzed by SA Biosciences web-based software (http://pcrdataanalysis.sabiosciences.com/pcr/arrayanalysis.php). The experimental results were calculated based on Quality control (QC) performance of housekeeping gene expression and statistical analysis. The changes in the gene expression levels were considered statistically significant with 35 PCR cycles (cut off value).

\subsubsection{Western blot analysis of LC3 and p62 (Sequestosome 1) in the stable} BHK-21/DENV2 replicon cells

The stable BHK-21/DENV2 replicon cells were cultured @ $5 \times 10^{5}$ cells in $60 \mathrm{~mm}$ dish supplemented DMEM with $10 \%$ FBS and incubated overnight at $37^{\circ} \mathrm{C}$ in a $\mathrm{CO}_{2}$ incubator. The cells were treated with $1.25 \mu \mathrm{M}$ of MPA for $24 \mathrm{~h}$. The stable BHK-21/DENV2 replicon cells without exposure to MPA was used as control. After the incubation, protein extracts were isolated from each group of cells using RIPA protein lysis buffer containing $1 \mathrm{mM}$ PMSF. Twenty microgram protein was separated on gel by $10 \%$ SDS-PAGE, transferred with polyvinylidene difluoride membrane (Merck; IPVH00010), blocked in 5\% BSA (Sigma, catalogue\#A1470) and probed independently with specific anti-LC3 (rabbit)(Thermo scientific; catalogue\#710014), anti-P62 (mouse)(Abcam, catalogue\# ab56416) and mouse anti $\beta$-actin (Thermo scientific, catalogue\#MA5-15739) antibodies against the target proteins. These were followed by incubation with corresponding Goat antiRabbit IgG (Sigma, catalogue\# A9169), anti-mouse IgG (Sigma; catalogue\#A9044) conjugated with HRP in separate set of experiments. Then the membrane was developed by adding the chemiluminescent WesternBright ECL developer (Advansta, catalogue\# K-12045-20) and the images were captured using with LI-COR C-DiGit Chemiluminescent Western Blot Scanner.The intensities of the protein bands were compared and analyzed using Image studio software (LiCor,C-Digit scanner). For all analysis, values of $\mathrm{P}<0.05$ were considered statistically significant.

\subsection{Effect of MPA on in vitro DENV2 infection}

\subsubsection{Treatment of MPA in DENV2 infected BHK-21 cells}

The BHK-21 cells were plated (@ $1 \times 10^{5}$ cells per well in 12 well tissue culture plates in DMEM with 10\% FBS and incubated overnight at $37^{\circ} \mathrm{C}$ in a $\mathrm{CO}_{2}$ incubator. On day- 0 , the media from the wells were aspirated and further infected with DENV2 (NGC strain passage over C6/36 cell line) @ 1 MOI in $400 \mu \mathrm{l}$ of DMEM with $2 \%$ FBS and 
incubated for $1 \mathrm{~h}$ at $37^{\circ} \mathrm{C}$ inside $\mathrm{CO}_{2}$ incubator, with mild rocking at 15 minutes intervals. After the incubation, the DENV2 inoculum was aspirated out and $2 \mathrm{~mL}$ DMEM was added per well. Subsequently, various MPA drug dosages $(0.078-5.0 \mu \mathrm{M})$ were added to the wells. The drug diluent, Dimethyl sulfoxide (DMSO) was used as the control treatment. The plates were then incubated overnight at $37^{\circ} \mathrm{C}$ in $\mathrm{CO}_{2}$ incubator for 24 hours. On day-1, $100 \mu \mathrm{l}$ of FBS was added to the wells to make up 5\% concentration and the incubation was continued. At the end of day-2, the culture media from wells were aspirated into $2 \mathrm{~mL}$ eppendorf tubes and centrifuged at $400 \mathrm{xg}$ for 10 minutes to remove the cell debris. The supernatants from different dosage treatments were transferred into fresh tubes and stored at $-80{ }^{\circ} \mathrm{C}$ until the plaque assays were done.

\subsubsection{Plaque assay}

$2 \times 10^{5}$ BHK-21 cells per well were plated in 24 well tissue culture plates in DMEM with $10 \%$ FBS. The plates were incubated overnight at $37^{\circ} \mathrm{C}$ in $\mathrm{CO}_{2}$ incubator to reach $100 \%$ confluent monolayer. The following day, DENV2 containing cell culture supernatants, from MPA dosage experiment, were serially diluted from $10^{-1}$ to $10^{-5}$ in serum free DMEM medium. The culture medium from each well was removed and the BHK-21 monolayer was washed with serum free DMEM to remove traces of serum. Further, $200 \mu \mathrm{l}$ of DENV2 serial dilutions of respective samples were added over the cells in the pre-designated wells. The plates were incubated for $1 \mathrm{~h}$ at $37^{\circ} \mathrm{C}$ inside $\mathrm{CO}_{2}$ incubator with mild rocking of plates at every 15 minutes intervals.

At $1 \mathrm{~h}$ post-incubation, the virus dilutions were removed and the wells were washed with serum free DMEM. Subsequently, $0.5 \mathrm{~mL}$ of overlay medium (DMEM with $0.8 \%$ carboxy methyl cellulose (EMD Millipore), $100 \mathrm{U} / \mathrm{ml}$ penicillin-streptomycin (Gibco) and 2\% FBS was added to the infected BHK-21 cells. The plates were incubated in the $\mathrm{CO}_{2}$ incubator at $37^{\circ} \mathrm{C}$ for 5 days, to progress the infection into cytopathic with the formation of plaques. At the end of incubation, the cells were fixed in $3 \%$ paraformaldehyde for 30 minutes and washed with tap water. The fixed cellular layer in the wells was stained with $1 \%$ crystal violet solution for 20 minutes. Excess stain was removed with tap water washing and the plates were dried. The number of unstained translucent plaques in the background of crystal violet stained cells indicated the number of infectious DENV2 particles present in the diluted samples. The numbers of plaques were converted to plaque forming units per $\mathrm{ml}$ of experimental samples using the equation given below. Virus titer $(\mathrm{pfu} / \mathrm{ml})=$ average number of plaques $/ 200 \mu \mathrm{l}$ inoculum x $1000 \mu \mathrm{l}$ x dilution factor.

\section{Results}

\subsection{Confirmation of in-vitro transcribed RNA (IVT-RNA)}

The pRS424 plasmid containing the sub-genomic DENV2 replicon was linearized with SacI Restriction Enzyme (RE) and separated on an agarose gel (Supplementary Fig. 1A). A single linear DNA band was observed upon RE digestion. The purified linear DNA (Supplementary Fig. 1B) was used as a template for in vitro RNA synthesis as described in the Methods. The IVT- RNA was separated and analyzed by agarose gel electrophoresis (Supplementary Fig. 1C).The purified IVT-RNA was transfected into naïve BHK-21 cells. The stable cells were selected by G418 antibiotic selection as detailed in Methods.

\subsection{MPA treatment shows minimal cytotoxicity on stable BHK-21/DENV2 replicon cells}

In vitro cytotoxicity of MPA on stable BHK-21/DENV2 replicon cells was examined by MTT assay. The optimum concentration (IC50 of MPA $=3.8 \mu \mathrm{M})$ was calculated as shown in the standard dose response curve (Supplementary Fig. 5).Based on MTT cytotoxicity assay, a sublethal dose of $1.25 \mu \mathrm{M}$ MPA was used for further analysis. The microscopic observation indicates that stable BHK-21/DENV2 replicon cells treated with $1.25 \mu \mathrm{M}$ MPA were morphologically similar to the untreated stable BHK-21/DENV2 replicon cells (Supplementary Fig. 2). The cell viability was found to be $90 \%$ in the untreated stable BHK-21/ DENV2 cells, whereas $82 \%$ cells were viable in the MPA-treated stable BHK-21/DENV2 cells by MTT assay.

\subsection{Stable BHK-21/DENV2 replicon cells show the expression of DENV2- NS4A}

The replication of DENV2 -RNA in stable BHK-21/DENV2 replicon cells was confirmed by the presence of DENV2 -NS 4A gene expression (Supplementary Fig. 3). The PCR amplified product of DENV2- NS4A gene corresponding to the band size of 855 bp in a $2 \%$ agarose gel indicates that there is an active replication of DENV2-RNA in the stable BHK-21/DENV2 replicon cells.

\subsection{MPA treatment inhibits DENV2-RNA replication as evidenced by reduction in Renilla Luciferase (RLuc) enzyme activity in cell lysates}

The effect of $1.25 \mu \mathrm{M}$ MPA treatment (24 hours) on DENV2-RNA replication was assessed in stable BHK-21/DENV2 replicon cells by measuring RLuc enzyme activity. The addition of MPA to stable BHK21/DENV2 replicon cells showed a significant reduction in Rluc activity (RLU) as compared to the untreated stable BHK-21/DENV2 replicon cells as represented in Fig. 1. The value of $p<0.05$ is statistically significant.

\subsection{MPA treatment inhibits DENV2- NS1 protein secretion into cell culture supernatants}

The semi-quantitative estimation of DENV2-NS1 protein in the

$$
\begin{gathered}
\text { RLuc Activity } \\
\mathrm{P}<0.05
\end{gathered}
$$

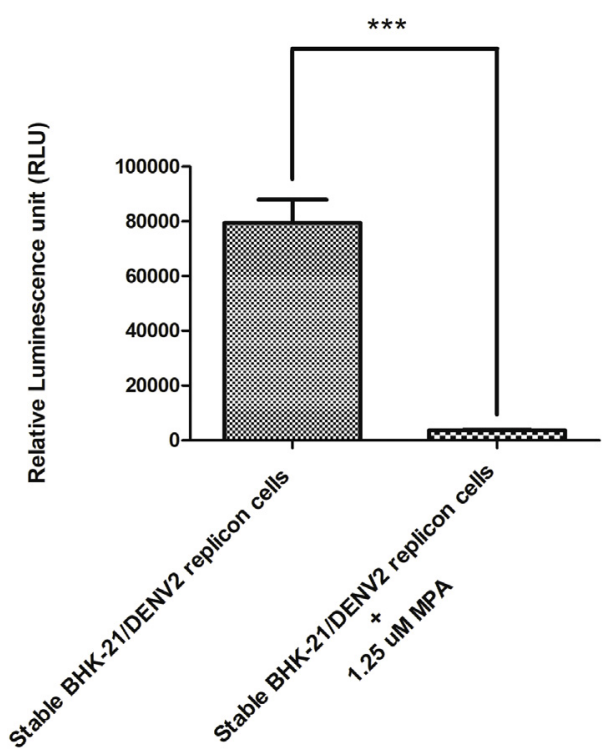

Fig. 1. Mycophenolic Acid (MPA) inhibits Renilla Luciferase (RLuc) enzyme activity in stable BHK-21/DENV2 replicon cells. Bar diagram showing RLuc enzyme activity of stable BHK-21/DENV2 replicon cells (untreated group) and 1.25 $\mu \mathrm{M}$ MPA (24 hours) treated stable BHK-21/DENV2 replicon cells (treated group). The relative RLuc activity was analyzed with unpaired Student's t-test (two-tailed, unequal variance) and graphs were made using Graph Pad Prism software. version 5.0. The data represent mean average value of three independent experiments $(n=3)+$ Standard Deviation $(\mathrm{p}<0.05)$. In the bar graph, $\mathrm{X}$-axis represents the stable BHK-21/DENV2 replicon cells \pm MPA and Y -axis represents RLuc enzyme units (RLU). 
NS1 secretion

$\mathrm{P}<0.05$

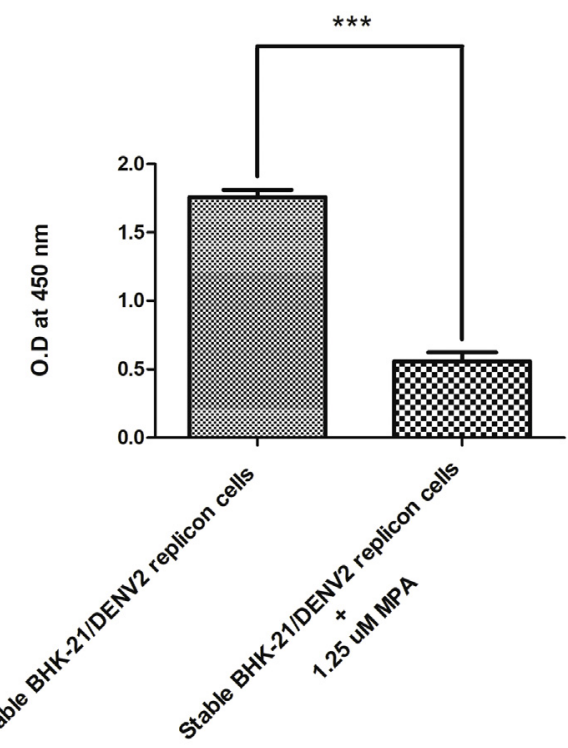

Fig. 2. Mycophenolic Acid (MPA) decreases the quantity of the secreted NS1 in stable BHK-21/DENV2 replicon cells. Histograms showing DENV2NS1 protein secretion from stable BHK -21/DENV2 replicon cells (untreated group) and 1.25 $\mu \mathrm{M}$ MPA (24 hours) treated stable BHK -21/DENV2 replicon cells (treated group).The optical densities (O.D) were measured spectophotometerically at $450 \mathrm{~nm}$. The data were analyzed with unpaired Student's t-test. The bar diagram was made using Graphpad Prism software version 5.0. The values $\mathrm{p}<0.05$ were statistically significant. In the bar graph $\mathrm{X}$-axis represents stable BHK-21/DENV2 replicon cells \pm MPA and $Y$-axis represents the O.D at $450 \mathrm{~nm}$ measured by ELISA.

culture supernatant of stable BHK-21/DENV2 replicon cells and the effect of $1.25 \mu \mathrm{M}$ MPA (24 hours) on its secretion was carried out by NS1 capture ELISA. The secretion of DENV2 -NS1 protein into the stable BHK-21/DENV2 replicon cell culture media was significantly reduced to $65 \%$ upon treatment with $1.25 \mu \mathrm{M}$ MPA in stable BHK-21/DENV2 replicon cells as compared to the untreated cells (Fig. 2). The value of $\mathrm{p}<0.05$ is statistically significant.

\subsection{MPA treatment alters the expression of ATGs in stable BHK-21/ DENV2 replicon cells}

3.6.1. Multi-gene expression analysis shows over-expression of initial autophagy genes in stable BHK-21/DENV2 replicon cells

Based on the above observation, further analysis was carried out to quantify the ATG expression at the transcriptional level in the stable BHK-21/DENV2 replicon cells treated with 1.25 $\mu \mathrm{M}$ MPA for 24 hours. Total RNA was extracted from MPA treated stable BHK-21/DENV2 cells, untreated cells and control cells (Naïve BHK-21). As shown in supplementary data (Excel sheet), the average threshold values (Ct) of all the ATGs obtained from the above three sets of untreated stable BHK-21/DENV2 replicon cells (Group 1), MPA-treated stable BHK-21/ DENV2 replicon cells (Group 2) and naïve BHK-21 cells were calculated. The relative expression level of the autophagy genes, which met the criteria of greater than 2-fold change was taken into consideration. The difference in the melt curves of autophagy genes represents the specificity of qRT-PCR amplified products.

The differential autophagy gene expression is represented by difference in color of heat maps (Fig. 3), which provides a visualization of the fold changes in gene expression in the context of the PCR array layout. Also, the differential expression of normalized autophagy-associated genes in stable BHK-21/DENV2 replicon cells (Group 1) and
MPA-treated BHK-21/DENV2 replicon cells (Group 2) are given in scatter plots (Fig. 4).

In the RT2 profiler, the expression of four genes, Unc-51 like kinase 2 (ULK2) (fold change =2.62), Cathepsin D (CTSD) (fold change $=2.07$ ), Trans-membrane protein (TMEM 74) (fold change $=7.32$ ) and Estrogen receptor protein 1 (ESR1) (fold change $=3.32$ ) were significantly higher in stable BHK-21/ DENV2replicon cells (Group 1) as shown in Fig. 8. It is known that proteins encoded by the above genes play a functional role in the initial induction of autophagy by the formation of pre-autophagosmal structures.

3.6.2. Multi-gene expression analysis shows downregulation of initial autophagy genes in stable BHK-21/DENV2 replicon cells upon MPA treatment

Next, we investigated the effect of MPA treatment on initial autophagy inducing genes which were seen upregulated in the stable BHK-21/DENV2replicon cells. The multi-gene analysis (Fig. 5) showed a downregulation of ULK2, CTSD, TMEM 74 and ESR1 autophagy genes in MPA treated stable BHK-21/DENV2 replicon cells (Fig. 5B) as compared to untreated stable BHK-21/DENV2 replicon cells (Fig. 5A). This result also indicates that the over-expression of autophagy initiator genes is dependent on the replication of dengue viral RNA in the stable BHK-2/DENV2 replicon cells (Fig. 6).

3.6.3. Cysteine protease coding genes were upregulated in stable BHK-21/ DENV2replicon cells upon MPA treatment

The up-regulation of ATG4A, ATG4B and ATG4C genes which code for cysteine proteases in MPA treated stable BHK-21/DENV2 replicon cells suggests that the MPA treatment activates cysteine proteases in stable BHK-21/DENV2 replicon cells. These cysteine proteases are involved in the de conjugation of lapidated LC3 (LC3II). In addition, AKT1, APP, ATG16L1, ATG16L2, B2M and HPRT1 genes were also upregulated in stable BHK-21/DENV2 replicon cells upon MPA treatment (Fig. 5). Overall, the expression of 10\% (10/84) of ATG genes were up-regulated greater than 2-fold in MPA treated stable BHK-21/ DENV2 replicon cells (Group 2) in RT2 Profiler PCR Array (Fig. 4).

\subsubsection{MPA treatment reduces LC3 lipidation (LC3 II)as well as p62 (SQSTM1)levels}

The measurement of LC3II and p62 protein levels are indicative substrates for the autophagic degradation and can be used to monitor autophagic flux. LC3 II is associated with the inner and outer membrane of autophagosomes whereas p62 binds to both ubiquitinated proteins and LC3. There was a reduction in LC3 II and p62 expression in the stable BHK-21/DENV2 replicon cells after MPA treatment as compared to the untreated cells (Fig. 7), indicating the degradation of autophagic substrates in autolysosomes. It is noteworthy that stable BHK-21/ DENV2 replicon cells harboring sustained replication of DENV2 subgenome showed an accumulation of LC3II as well as p62 proteins. This observation further indicates an important role of these proteins for the effective replication of the DENV2 subgenome without undergoing the fusion and degradation.

\subsubsection{MPA inhibits DENV2 multiplication}

The treatment of MPA with DENV2 infected BHK-21 cells as detailed in the methods (2.6.1), showed significant inhibition of dengue virus titer in the cell culture supernatant as compared to untreated BHK21 cells. The analysis revealed that MPA treated BHK-21 cells exhibited 99-100\% clearance of infection at concentrations greater than $1.25 \mu \mathrm{M}$ MPA $(\mathrm{n}=3)$ at an MOI of 1 . The control treatment (DMSO, 1 MOI) had $2.75 \times 10^{5} \mathrm{PFU} / \mathrm{mL}$ of DENV2 infectious particles, whereas when treated with $1.25 \mu \mathrm{M}$ concentration of MPA, the number of infectious particles were reduced to $2000 \mathrm{PFU} / \mathrm{mL}$. Dose-response curves of MPA treatment versus \% DENV2 inhibition demonstrated that there was $99.9 \%$ of DENV2 inhibition at $1.25 \mu \mathrm{M}$ MPA. An exponential fit of the 
(A)

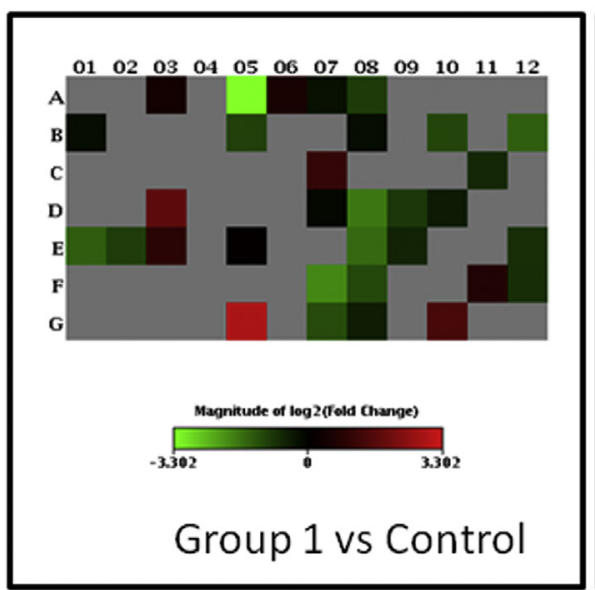

(B)

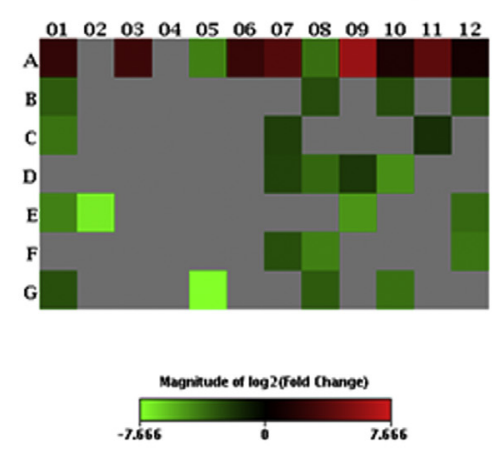

Group 2 vs Control
(C)

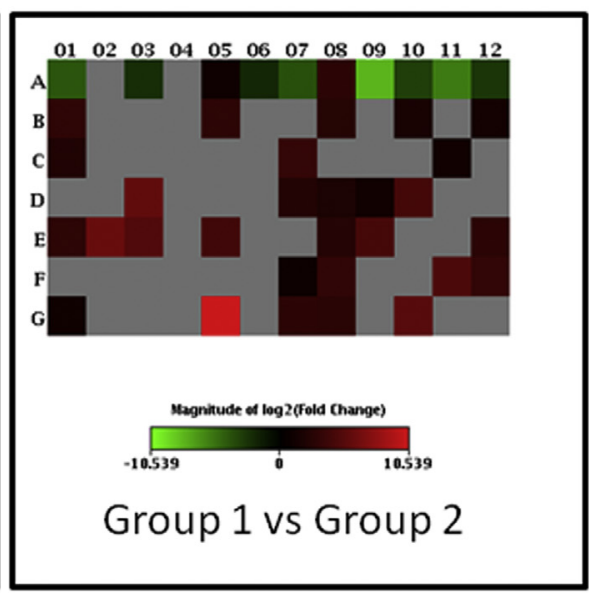

Fig. 3. Heat maps showing differential expression of autophagy-associated genes. Based on analysis of RT2 Profiler PCR array, heat maps show differential expression of autophagy-associated genes in three groups (A, B and C). (A) Group 1 versus Control (B) Group 2 versus Control (C) Group 1 versus Group 2. The fold change greater than 2 is highlighted in red, less than 2 is in green and unchanged genes in black. The heat map represents a visualization of the fold changes in the context of the array layout. Stable BHK-21/DENV2 replicon cells (Group 1), BHK-21/DENV2 replicon cells treated with MPA (1.25 $\mu$ M) for 24 hours (Group 2) normalized with control group (naïve BHK-21 cells).

(A)

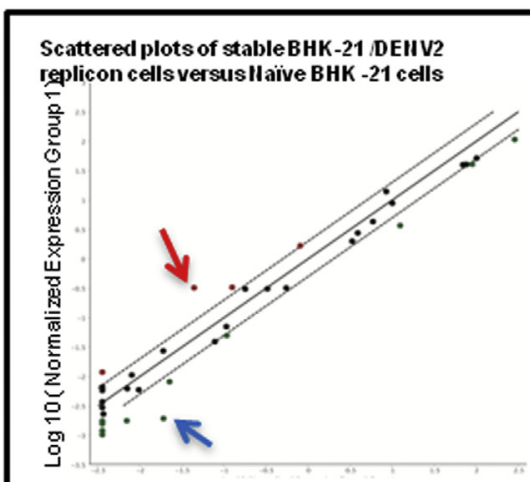

Log 10 (NormalizedExpression Control Group)
(B)

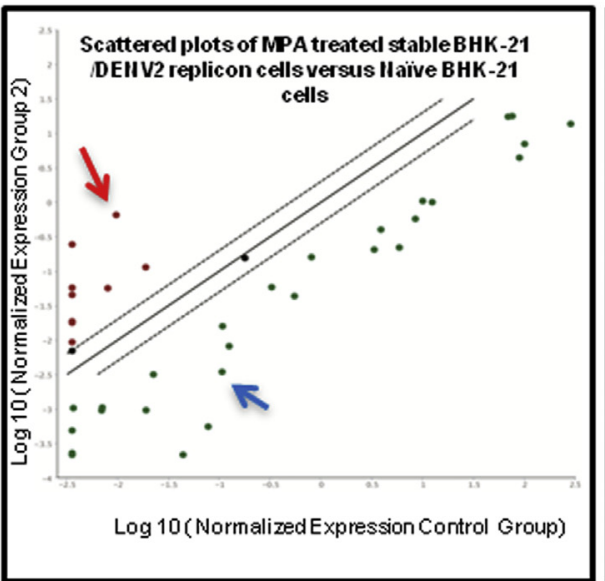

(C)

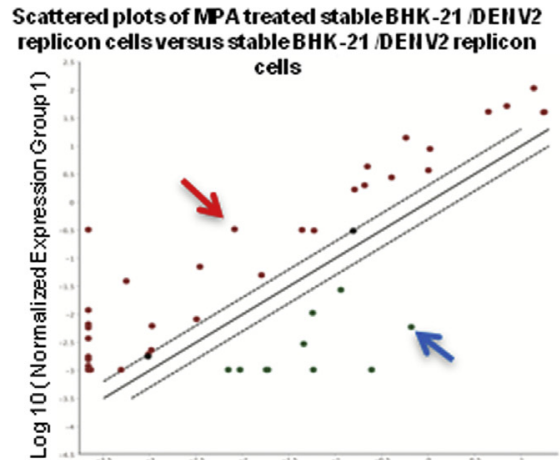

Log 10 (Normalized Expression Group 2)

Fig. 4. Scatter plots showing the differential expression of autophagy-associated genes. (A) Group 1 versus control group; (B) Group 2 versus control group; (C) Group 1 versus Group 2. Based on analysis of RT2 Profiler PCR array, scatter plots displayed differential expression of autophagy-associated genes in three groups (A,B and C) with fold change greater than 2.The red arrow indicates gene upregulation and blue arrow indicates downregulation. Upregulated genes greater than 2 were highlighted in brown, downregulated less than 2 were in green and unchanged genes in black. Stable BHK-21/DENV2 replicon cells (Group 1), stable BHK-21/ DENV2 replicon cells treated with $1.25 \mu \mathrm{M}$ MPA for 24 hours (Group 2) and control group (naïve BHK -21 cells).

$\log$ [drug concentration] versus DENV2 infectious particles per various dosage treatments (Fig. 8) showed that $0.293 \mu \mathrm{M}$ MPA can inhibit $50 \%$ of infection (EC50).

\section{Discussion}

The proviral and/or antiviral role of cellular autophagy within the infected cells vary depending on the viral tropism $[8,18,19,20]$. Accumulating evidence suggests that DENV infection induces autophagy vesicles, which aid in the viral replication and protect from the host immune system [21]. The effective role of antiviral drugs such as MPA on DENV inhibition is poorly studied. In the present study, the stable BHK-21/DENV2 replicon cells were generated which exhibited notable autophagy gene expression changes induced by sub-genomic DENV2 replicon RNA. The present data provide an interesting insight into autophagy induction in these cells and the inhibitory effect of MPA on it. Furthermore, in the stable BHK-21/DENV2 replicon cells, dengue viral subgenomic DENV2 RNA is expressed at levels similar to the cells infected with DENV2 (NGC strain) RNA [14]. The presence of DENV2NS4A gene amplification in stable BHK-21/DENV2 replicon cells indicates the effective DENV2 -RNA replication. Understanding dengue viral inhibition as well as activation of host genes and their coordination by cell signaling processes in presence of viral inhibitors is an attractive strategy to design antiviral drug analogues to curb DENV infection. There is a paucity of literature to specify the action of MPA in dengue viral replication. 

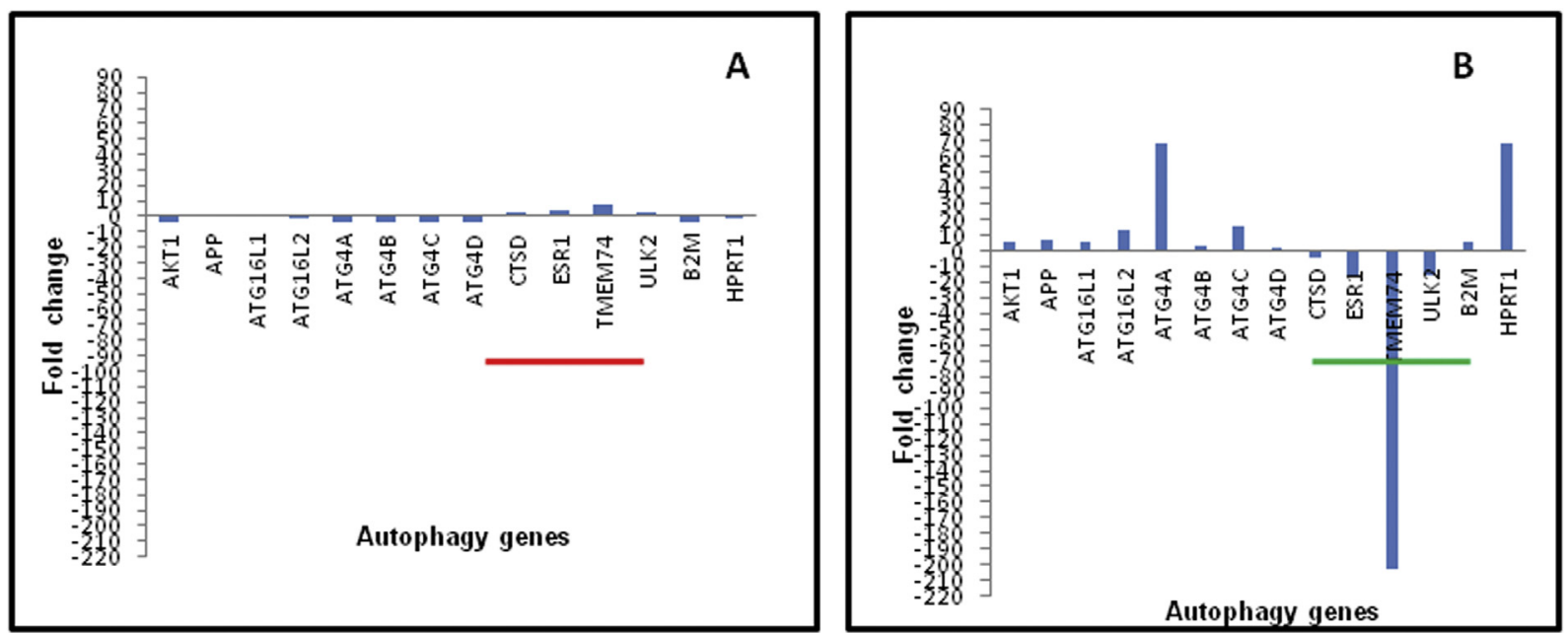

Fig. 5. Multi gene analysis displaying the relative autophagy gene expression levels. (A) Increased or decreased gene regulation in stable BHK-21/DENV2 replicon cells (untreated group) (B) Altered gene expression in stable BHK 21/DENV2 replicon cells treated with 1.25 $\mu$ M MPA for 24 hours (treated group). X-axis indicates autophagy genes, Y-axis indicates fold change in gene expression. The upregulated genes CTSD (fold change 2.074), ESR1 (fold change 3.32), TMEM74 (fold change 7.32) and ULK2 (fold change 2.62) are underlined with red colour in (A). The downregulated genes upon MPA treatment are underlined with green colour in (B).

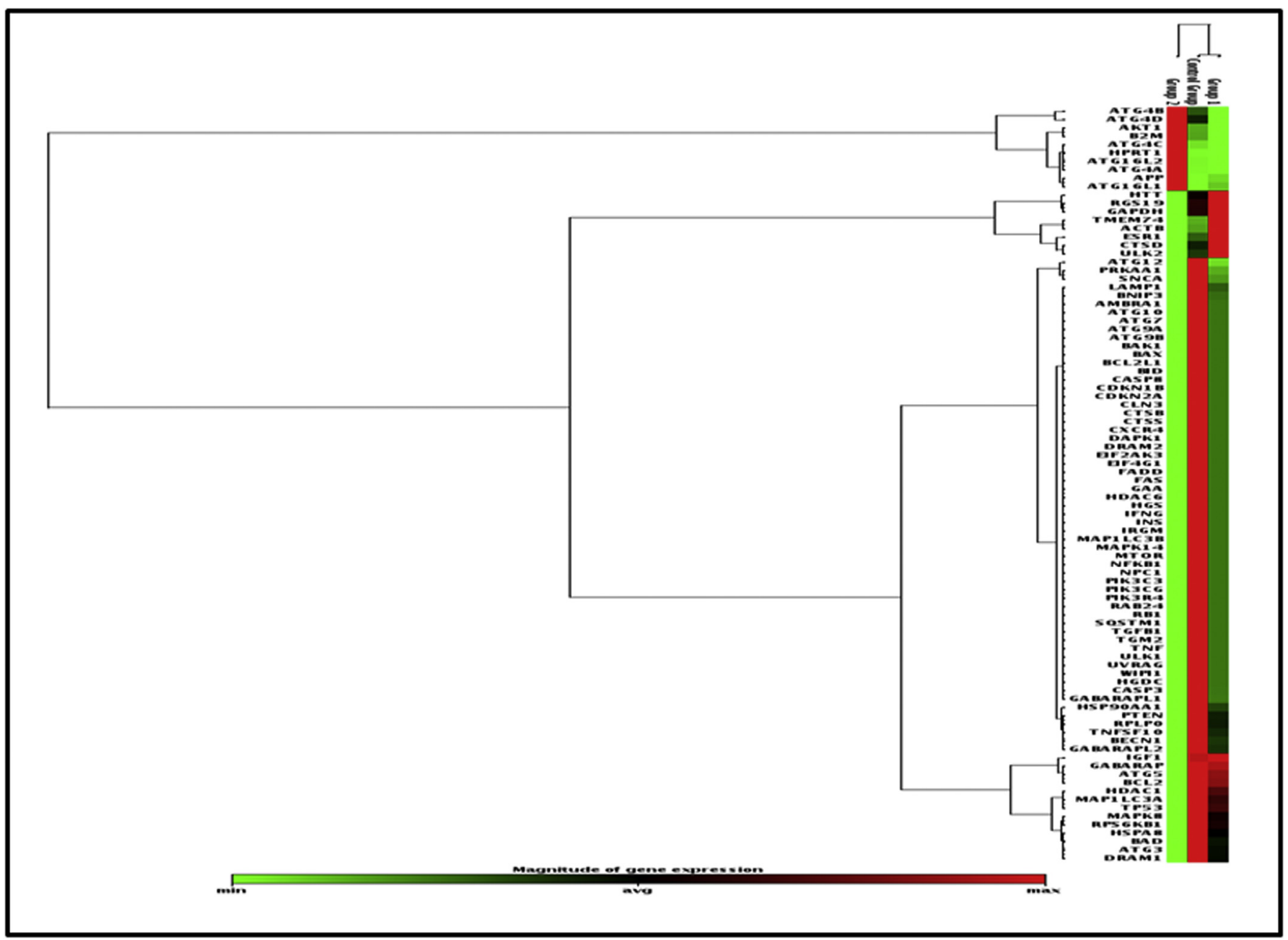

Fig. 6. A clustergram of the expression profile of autophagy genes. Relative expression patterns of autophagy-associated genes in stable BHK -21/DENV2 replicon cells (Group1), 1.25 $\mathrm{M}$ MPA (24 hours) treated BHK-21/DENV2 replicon cells (Group 2) and control group (naïve BHK-21), as shown in a clustergram based on RT2 Profiler PCR array (PAHS-084Z). Expression clusters were indicated by color bars next to the genes and groups that were included in different clusters. The magnitude of gene expression scale is indicated at the bottom, which represents the gene relative expression levels by a range of colors. The clustergram represents hierarchical clustering of the entire co-regulated genes across the three groups. 


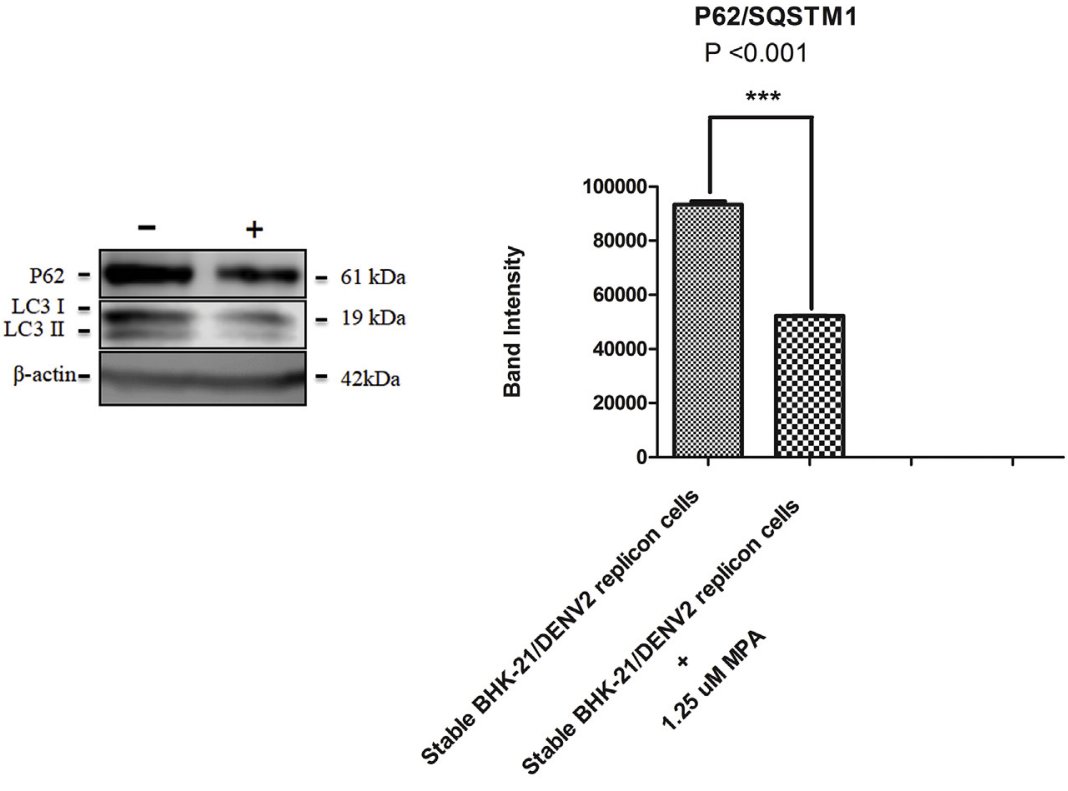

(A)

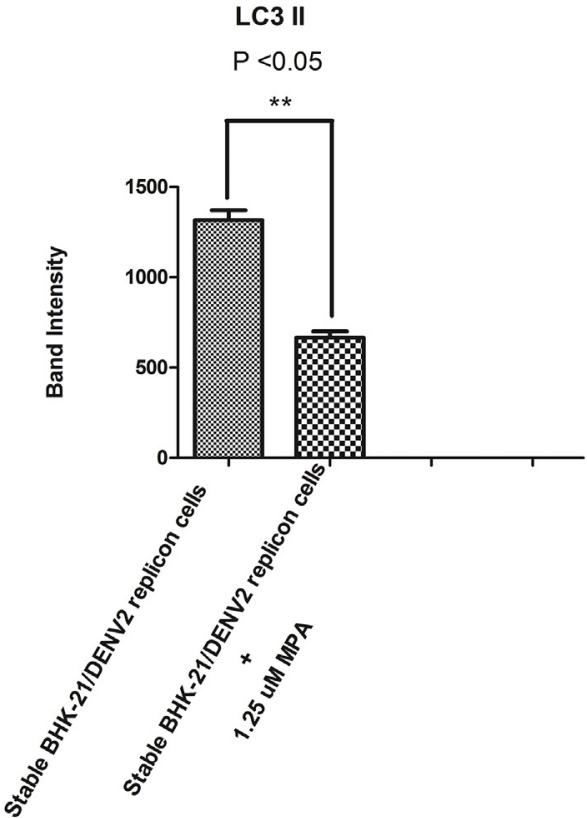

(B)

Fig. 7. LC3 and p62 (Sequestosome 1) in MPA untreated/treated stable BHK-21/DENV2 replicon cell lysates. Stable BHK-21/DENV2 replicon cells were treated with ( + ) and without ( - ) $1.25 \mu \mathrm{M}$ MPA (24 hours). Approximately $20 \mu \mathrm{g}$ of protein was separated on a gel by $10 \%$ SDS-PAGE, transferred to $0.45 \mu \mathrm{m}$ PVDF membrane and blocked in 5\% BSA in TBST. The membranes were probed with 1:1000 dilution of Rabbit anti-LC3 antibody (Thermo scientific; 710014), mouse antiP62 (Abcam; ab56416), and mouse anti $\beta$-actin (Thermo scientific; MA5-15739) were detected with the appropriate secondary antibodies using chemiluminescent WesternBright ECL developer (Advansta; K-12045-20). The band intensities of p62 protein expression level (A) and LC3II (B) between MPA treated and untreated stable BHK-21/DENV2 replicon cells were carried out using Image studio software (LiCor,C-Digit scanner) and GraphPad Prism software version 5.0. Data from western blotting were analyzed with unpaired Student's t-test (two-tailed, unequal variance). For all analysis, values of $\mathrm{p}<0.05$ were considered statistically significant.

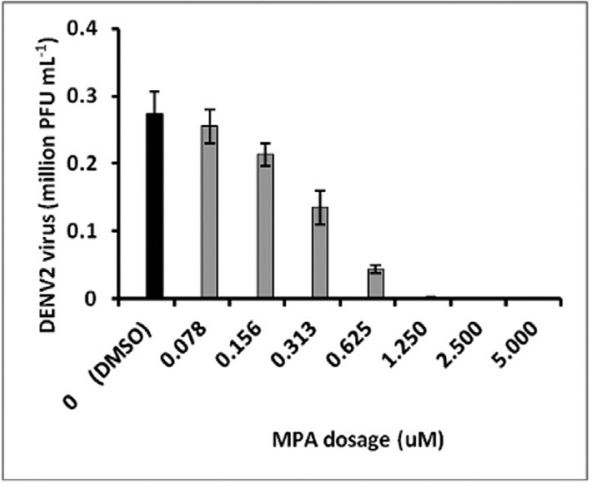

(A)

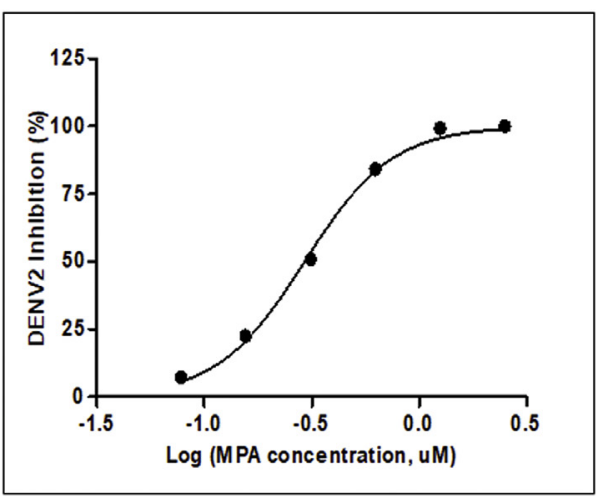

(B)
Fig. 8. MPA inhibits DENV2 virus titer in the cells infected with DENV2 (NGC strain). (A) BHK-21 cells were infected with 1 MOI of DENV2 (NGC strain) and subjected to the treatment of various concentrations of MPA ranging from $0.078 \mu \mathrm{M}$ to $5 \mu \mathrm{M}$ and incubated for 24 hours. DENV2 titers were measured in the cell culture supernatant in treated and untreated cells by plaque assay. The data is expressed in Plaque Forming Units (PFU) per ml. (B) Dose response curve of MPA versus virus yield in the cell culture supernatant of DENV2 (NGC strain) infected BHK21 cells. $\mathrm{X}$-axis represents concentration of MPA as $\log (\mu \mathrm{M})$; Y-axis represents the percent inhibition of DENV2 yield. MPA treatment inhibits $50 \%$ of infection (EC50) at the concentration of $0.293 \mu \mathrm{M}$.
In this context, the effect of MPA was tested on the stable BHK-21/ DENV2 replicon cells and the expression of p62 and LC3II substrates involved in autophagosome degradation. Additionally, autophagy gene expression under the influence of MPA was analyzed in stable BHK-21/ DENV2 replicon cells. The drug concentration of MPA for treating stable BHK-21/DENV2 replicon cells was selected based on EC50 value of dose response curve (Supplementary Fig. 5) in MTT assay. The recombinant plasmid pRS424/DENV2 replicon construct contains Renilla luciferase gene upstream of DENV2 nonstructural genes under the control IRES. Therefore, expression of luciferase enzyme activity is directly proportional to the replication/translation of DENV2 sub-genome in stable BHK-21/DENV2 replicon cells. Upon treatment of stable BHK21/DENV2 replicon cells with MPA showed a significant decrease in the Luciferase enzyme activity indicating the inhibition of DENV2 RNA replication/translation. Similarly, the treatment of MPA reduced the level of DENV2-NS1 protein in the cell culture supernatant of stable BHK-21/DENV2, supporting the aforementioned decrease in the luciferase activity. These observations, further suggests that MPA may transiently halt the replication process without effecting the cell viability of the stable BHK-2/DENV2 replicon cells.

We noted a significant alteration in host autophagy gene expression after the MPA treatment of stable BHK- 21/DENV2 replicon cells. Such a change in gene expression pattern provides critical, cumulative information on the functional role of autophagy flux under stress conditions, which often correlates with DENV replication in the infected host cells. The relative autophagy gene expression profile in vitro observed here offers some interesting cues as to how dengue virus replication might be affecting this process in vivo and also how DENV is hijacking the host translation machinery and multiplying in the infected cells. In Hepatitis C virus (HCV), autophagy helps in the initial gene translation 
but not in the maintenance of viral replication [19]. The Measles virus induces the earlier event of autophagy by binding to the cell surface receptor CD46 facilitating its viral entry [18]. There is also evidence, which suggests that unlike enteroviruses, DENV infection depends on host machinery for making dengue viral envelope proteins via lipophagy, an autophagy dependent process activated in replication of DENV [22].

In the present study, we noted upregulation of ULK2, CTSD, ESR1 and TMEM74 expression in the stable BHK-21/DENV2 replicon cells with actively replicating sub-genomic DENV2- RNA. These genes were downregulated in the post MPA treatment. Based on our results, it is conceivable that these genes play a major role in dengue virus replication via activating initial autophagy machinery leading to formation of pre-autophagosomal structure (PAS), which gets halted upon the MPA treatment. To the best of our knowledge, this is the first report demonstrating relative autophagy gene expression profile in stable BHK-21/DENV2 replicon cells and the effect of MPA on it. The proteins encoded by the upregulated genes in stable BHK-21/DENV2 replicon cells perform important cellular functions in the autophagy induction process. The upregulated ULK2 gene is crucial for the autophagy initiation [23] and Cathepsin D (CTSD) is an important autophagy marker [5] in DENV replication. Inhibition of CTSD impairs the autophagy flux and TMEM74 protein over-expression has a role in the autophagy cellular process [24]. Also, TMEM 74 is involved in the initial stage of functional autophagosome formation [24].

The MPA treatment shows an aberrant expression of ATGs in stable BHK-21/DENV2 replicon cells including the upregulation of ATG4A, ATG4B, ATG4C, ATG4D, AKT1, APP, ATG16L1, ATG16L2, B2M and HPRT1 genes. The ATG4A, ATG4B and ATG4C code for Cysteine proteases involved in conjugation of LC3 to LC3-PE [25] as well as de conjugation of LC3-PE to LC3 [26]. These cysteine proteases, which are upregulated in MPA treated stable BHK-21/DENV2 cells are probably involved in deconjugation of LC3-PE to LC3. The significant upregulation of HPRT1 gene expression observed here suggests the perturbation in purine nucleotide metabolism. It is reported that MPA inhibits IMPDH (Inosine Monophosphate Dehydrogenase) activity resulting in the depletion of GTP pool and inhibition of DENV replication [15]. Therefore, the enhancement in the HPRT1 gene expression may induce a positive modulation of salvage pathway of purine metabolism. However, the replication could not be rescued, most likely due to downregulation of the early phase autophagy genes, and upregulation of cysteine protease coded by ATG genes. The increase in the expression of ULK2, CTSD, ESR1 and TMEM74 genes in stable BHK-21/DENV2 replicon cells suggest that DENV2 replication depends on early phase of autophagosome formation which helps in DENV2 replication. In addition, our data are in accordance with the previous report where the virus induced an initial activation of autophagic flux during infection [27].

There is decrease in LC3 II (lapidated LC3) and p62 (Sequestosome 1) protein expression in MPA treated stable BHK-21/DENV2 replicon cells in comparison to the untreated cells. LC3 II and p62 are autophagy substrates and get degraded upon fusion of autophagosome with lysosome [28]. Therefore, it can be surmised that MPA inhibits DENV2 genome replication by promoting the degradation of autophagosome along with the enhancement of ATG4A gene expression resulting in the delipidation of LC3II [28]. Further, it was observed that MPA treatment suppresses ATGs involved in the PAS formation. It's also noteworthy that MPA accelerates the autophagic degradation process as evidenced by the significant upregulation of HPRT1, an enzyme involved in the salvage pathway of purine nucleotide. This observation is in agreement with an earlier study which showed that MPA inhibits de-novo synthesis of guanine nucleotide by targeting IMPDH (IMP dehydrogenase) [29]. In the in vitro system, DENV2 (NGC strain) multiplication was significantly reduced upon the treatment with MPA as indicated by the reduction in virus titer (plaque assay) in the DENV2 infected BHK21 cells. Our data shed light on DENV pathogenesis and prompt further investigation into the design of antiviral drug therapy with respect to the autophagy induction.

\section{Conclusion}

Current study highlights that MPA can effectively inhibit DENV2 subgenomic RNA replication and alter the autophagic degradation as evidenced by the decrease in $\mathrm{p} 62$ and LC3II protein levels. Importantly, there was an upregulation of ATG4A gene expression which codes for cysteine protease involved in the delipidation of LC3II. We also observed that MPA suppresses the ATGs responsible for the formation of PAS, which are found to be elevated during the sustained replication of DENV2 subgenomic RNA. Further, MPA was enhancing the expression of HPRT1 gene indicating the possibility of utilizing salvage of purine nucleotides. Notably, the inhibitory effect of MPA in reducing the viral titer of DENV2 in the infected BHK-21 cells was clearly evident. Overall, our findings are of critical significance in understanding the molecular pathogenesis of dengue virus with respect to modulation of autophagy. Our study provides further impetus in designing new MPA analogues to effectively inhibit dengue viral replication and to a better understanding of host-virus interactions.

\section{Funding}

RP acknowledges Central University of Kerala, Govt of India for providing the infrastructural facilities. This work was supported by Department of Science and Technology (DST)- Science and Engineering Research Board (SERB), Young Scientist Scheme (SB/YS/LS-366/2013), Govt of India, New Delhi and Kerala State Council for Science, Technology \& Environment (KSCSTE)-Kerala Biotechnology Commission (KBC) under Young Investigator Scheme (015/YIPBIKBC/ 2013/CSTE), Govt of Kerala, India. RD was supported by Rajiv Gandhi National Fellowship (RGNF-2012-13-ST-ORI-30084), Govt of India.

\section{Declaration of competing interest}

The authors had no conflict of interest to declare.

\section{Acknowledgements}

We thank Prof. Padmanabhan (Georgetown University, USA) for providing pRS424-DENV2 replicon plasmid as a kind gift for our study.

\section{Appendix A. Supplementary data}

Supplementary data to this article can be found online at https:// doi.org/10.1016/j.micpath.2019.103762.

\section{References}

[1] S.B. Halstead, Dengue, Lancet 370 (9599) (2007) 1644-1652.

[2] Y.W. Wu, C. Mettling, S.R. Wu, C.Y. Yu, G.C. Perng, Y.S. Lin, Y.L. Lin, Autophagyassociated Dengue Vesicles Promote Viral Transmission Avoiding Antibody Neutralization Scientific Reports vol. 6, (2016), p. 32243.

[3] B. Levine, D.J. Klionsky, Development by self-digestion: molecular mechanisms and biological functions of autophagy, Dev. Cell 6 (4) (2004) 463-477.

[4] C. Grose, Autophagy during common bacterial and viral infections of children, Pediatr. Infect. Dis. J. 29 (11) (2010) 1040-1042.

[5] M. Panyasrivanit, M.P. Greenwood, D. Murphy, C. Isidoro, P. Auewarakul, D.R. Smith, Induced autophagy reduces virus output in dengue infected monocytic cells, Virology 418 (2011) 74-84.

[6] N.S. Heaton, R. Perera, K.L. Berger, S. Khadka, D.J. Lacount, R.J. Kuhn, G. Randall, Dengue virus nonstructural protein 3 redistributes fatty acid synthase to sites of viral replication and increases cellular fatty acid synthesis, Proc. Natl. Acad. Sci. 107 (2010) 17345-17350.

[7] Y.R. Lee, H.Y. Hu, S.H. Kuo, H.Y. Lei, Y.S. Lin, T.M. Yeh, C.C. Liu, H.S. Liu, Dengue virus infection induces autophagy: an in vivo study, J. Biomed. Sci. 20 (2013) 65.

[8] W.T. Jackson, T.H. Giddings Jr., M.P. Taylor, S. Mulinyawe, M. Rabinovitch, R.R. Kopito, K. Kirkegaard, Subversion of cellular autophagosomal machinery by RNA viruses, PLoS Biol. 3 (5) (2005). 
[9] S.B. Kudchodkar, B. Levine, Viruses and autophagy, Rev. Med. Virol. 19 (6) (2009) 359-378.

[10] Jeffrey E. McLean, Aleksandra Wudzinska, Emmanuel Datan, Daniela Quaglino, Zahra Zakeri, Flavivirus NS4A-induced autophagy protects cells against death and enhances virus replication, J. Biol. Chem. 286 (2011) 22147-22159.

[11] Y.R. Lee, H.Y. Lei, M.T. Liu, J.R. Wang, S.H. Chen, Y.F. Jiang-Shieh, Y.S. Lin, T.M. Yeh, C.C. Liu, H.S. Liu, Autophagic machinery activated by dengue virus enhances virus replication, Virology 374 (2008) 240-248.

[12] E. Datan, S.G. Roy, G. Germain, N. Zali, J.E. McLean, G. Golshan, S. Harbajan, R.A. Lockshin, Z. Zakeri, Dengue-induced autophagy, virus replication and protection from cell death require ER stress (PERK) pathway activation, Cell Death Dis. 7 (2016).

[13] B.J. Thomson, Viruses and apoptosis, Int. J. Exp. Pathol. 82 (2001) 65-76.

[14] Chuan Young Ng, Feng Gu, Wai Yee Phong, Yen-Liang Chen, Siew Pheng Lim, Andrew Davidson, G. Subhash, Vasudevan, Construction and characterization of a stable subgenomic dengue virus type 2 replicon system for antiviral compound and siRNA testing, Antivir. Res. 76 (2007) 222-231.

[15] Ratree Takhampunya, Sukathida Ubol, Huo-Shu Houng, Craig E. Cameron, Radhakrishnan Padmanabhan, Inhibition of dengue virus replication by mycophenolic acid and ribavirin, J. Gen. Virol. 87 (2006) 1947-1952.

[16] M.S. Diamond, M. Zachariah, E. Harris, Mycophenolic acid inhibits dengue virus infection by preventing replication of viral RNA, Virology 304 (2002) 211-221.

[17] M.S. Diamond, D. Edgil, T.G. Roberts, B. Lu, E. Harris, Infection of human cells by dengue virus is modulated by different cell types and viral strains, J. Virol. 74 (17) (2000) 7814-7823.

[18] P.E. Joubert, G. Meiffren, I.P. Grégoire, G. Pontini, C. Richetta, M. Flacher, O. Azocar, P.O. Vidalain, M. Vidal, V. Lotteau, P. Codogno, C. Rabourdin-Combe, M. Faure, Autophagy induction by the pathogen receptor CD46, Cell Host Microbe 6 (4) (2009) 354-366.

[19] M. Dreux, P. Gastaminza, S.F. Wieland, F.V. Chisari, The autophagy machinery is required to initiate hepatitis $\mathrm{C}$ virus replication, Proc. Natl. Acad. Sci. U.S.A. 106
(33) (2009) 14046-14051.

[20] A. Schlegel, T.H. Giddings Jr., M.S. Ladinsky, K. Kirkegaard, Cellular origin and ultrastructure of membranes induced during poliovirus infection, J. Virol. 70 (10) (1996) 6576-6588.

[21] Erica L. Sanchez, Michael Lagunoff, Viral activation of cellular metabolism, Virology 479 (2015) 609-618.

[22] N.S. Heaton, G. Randall, Dengue virus-induced autophagy regulates lipid metabolism, Cell Host Microbe 8 (5) (2010) 422-432.

[23] Ryan C. Russell, Hai-Xin Yuan, Kun-Liang Guan, Autophagy regulation by nutrient signaling, Cell Res. 24 (2014) 42-57.

[24] C. Yu, L. Wang, B. Lv, Y. Lu, L. Zeng, Y. Chen, D. Ma, T. Shi, L. Wang, TMEM74, a lysosome and autophagosome protein, regulates autophagy, Biochem. Biophys. Res. Commun. 369 (2008) 622-629.

[25] Lan Zhang, Jingjing Li, Liang Ouyang, Bo Liu, Yan Cheng, Unraveling the roles of Atg 4 proteases from autophagy modulation to targeted cancer therapy, Cancer Lett. 373 (2016) 19-26.

[26] Zhong-Qiu Yu, Tao Ni, Bing Hong, Hai-Yan Wang, Fen-Jun Jiang, Shenshen Zou, Yong Chen, Dual roles of Atg8 - PE deconjugation by Atg4 in autophagy, Autophagy 8 (2012) 883-892.

[27] Metz Philippe, Abhilash Chiramel, Laurent Chatel-Chaix, Gualtiero Alvisi, Bankhead Peter, Rodrigo Mora-Rodríguez, Gang Long, Anne Hamacher-Brady, Nathan R. Brady, Ralf Bartenschlager, Dengue virus inhibition of autophagic flux and dependency of viral replication on proteasomal degradation of the autophagy receptor p62, J. Virol. 89 (2015) 8026-8041.

[28] T. Maruyama, N.N. Noda, Autophagy-regulating protease Atg4: structure, function, regulation and inhibition, J. Antibiot. (Tokyo) 71 (1) (2017) 72-78.

[29] W. Markland, T.J. McQuaid, J. Jain, A.D. Kwong, Broad-spectrum antiviral activity of the IMP dehydrogenase inhibitor VX-497: a comparison with ribavirin and demonstration of antiviral additivity with alpha interferon, Antimicrob. Agents Chemother. 44 (4) (2000) 859-866. 